\title{
Fast and accurate determination of the Wigner rotation matrices in the fast multipole method
}

\author{
Holger Dachsel ${ }^{\mathrm{a})}$ \\ John von Neumann Institute for Computing, Central Institute for Applied Mathematics, \\ Research Centre Jülich, 52425 Jülich, Germany
}

(Received 20 December 2005; accepted 16 March 2006; published online 14 April 2006)

\begin{abstract}
In the rotation based fast multipole method the accurate determination of the Wigner rotation matrices is essential. The combination of two recurrence relations and the control of the error accumulations allow a very precise determination of the Wigner rotation matrices. The recurrence formulas are simple, efficient, and numerically stable. The advantages over other recursions are documented. (C) 2006 American Institute of Physics. [DOI: 10.1063/1.2194548]
\end{abstract}

\section{INTRODUCTION}

In several scientific applications such as molecular dynamics $^{1}$ and plasma physics ${ }^{2}$ the evaluation of a pairwise potential is required. Very often this is the most time consuming step in a calculation. The direct method to evaluate these potentials scales quadratically with the number of particles $N$ which places a severe restraint on the size of systems which can be treated. One of the methods to achieve linear scaling is Greengard's fast multipole method (FMM) ${ }^{3,4}$ The FMM expands local charges in multipole expansions. The fast multipole method can be applied to the evaluation of $r^{-n}(n>0)$ pairwise interactions.

The implementation of the FMM to treat point charges in a very efficient way is the first step towards the continuous fast multipole method (CFMM) to calculate charge distributions arising in density functional and Hartree-Fock calculations. The ideas of FMM can be applied to the evaluation of electron repulsion integrals (ERI's). The computation of the ERI's is in general a step which requires $O\left(N^{4}\right)$ work regarding the number of basis functions $N$. By several computational techniques ${ }^{5}$ the scaling could be improved significantly to $O\left(N^{2}\right)$. The use of CFMM gives the possibility to make a further improvement in scaling, from $O\left(N^{2}\right)$ to $O(N)$. The Coulomb interaction of two charge distributions decreases exponentially with increasing separation, and the two distributions then interact as classical point charges.

An important modification of the FMM leads to the rotation based FMM (Ref. 6) which has a better scaling with regard to the length of the multipole expansions compared to the conventional FMM. The rotations are based on the Wigner rotation martices. The accurate calculation of these matrices is a requirement for a high accuracy of the rotation based FMM. Elliott and Board ${ }^{7}$ have proposed a fast fourier transform accelerated fast multipole algorithm. The scaling of this approach is better compared to the scaling of the rotation based FMM but additional approximation is involved, and therefore, the FMM error bounds are not retained.

\footnotetext{
${ }^{a)}$ Electronic mail: h.dachsel@fz-juelich.de
}

\section{THEORY}

The conventional fast multipole method requires $O\left(p^{4}\right)$ work with regard to the length of the multipole expansions $p$. $O\left(p^{3}\right)$ scaling can be achieved by rotation of the coordinate system to a position where the chargeless moments $O_{l m}$ and $M_{l m}$ take an explicitly simple form, ${ }^{6,8}$

$$
\begin{aligned}
& O_{l m}(a, 0,0)=\frac{a^{l}}{l !} \delta_{m 0}, \\
& M_{l m}(r, 0,0)=\frac{l !}{r^{l+1}} \delta_{m 0} .
\end{aligned}
$$

$\delta_{m k}$ is the Kronecker delta,

$$
\delta_{m k}= \begin{cases}0, & m \neq k \\ 1, & m=k .\end{cases}
$$

In standard presentations the three Euler angles are required to perform a rotation. In our case we require only two rotations, the first about the $Z$ axis followed by a rotation about the $Y$ axis. The multipole moments $\omega_{l m}$ with respect to the rotated coordinate system are linear combinations of the multipole moments $\omega_{l k}^{\prime}$ with respect to the original coordinate system,

$$
\omega_{l m}=\sum_{k=-l}^{l} \sqrt{\frac{(l-k) !(l+k) !}{(l-m) !(l+m) !}} d_{m k}^{l}(\theta) e^{-i k \phi} \omega_{l k}^{\prime} .
$$

The coefficients of the Taylor expansions are rotated similary,

$$
\mu_{l m}=\sum_{k=-l}^{l} \sqrt{\frac{(l-m) !(l+m) !}{(l-k) !(l+k) !}} d_{m k}^{l}(\theta) e^{i k \phi} \mu_{l k}^{\prime} .
$$

Equations (4) and (5) give the following relation:

$$
\sum_{m=-l}^{l} \omega_{l m} \mu_{l m}=\sum_{m=-l}^{l} \omega_{l m}^{\prime} \mu_{l m}^{\prime} .
$$

The associated Legendre polynomials included in the multipole moments and in the coefficients of the Taylor expansions can be rotated separately, 
TABLE I. Maximum absolute and relative errors of the computation of the $d_{k m}^{l}(\pi / 2)$.

\begin{tabular}{|c|c|c|c|c|c|c|}
\hline \multirow[b]{2}{*}{$p$} & \multicolumn{2}{|c|}{ First recursion relation } & \multicolumn{2}{|c|}{ Second recursion relation } & \multicolumn{2}{|c|}{ Both recursion relations } \\
\hline & $\Delta_{d, \text { absolute }}(p)$ & $\Delta_{d, \text { relative }}(p)$ & $\Delta_{d, \text { absolute }}(p)$ & $\Delta_{d, \text { relative }}(p)$ & $\Delta_{d, \text { absolute }}(p)$ & $\Delta_{d, \text { relative }}(p)$ \\
\hline 10 & $1.3 \times 10^{-13}$ & $1.3 \times 10^{-10}$ & $4.4 \times 10^{-15}$ & $1.2 \times 10^{-13}$ & $2.8 \times 10^{-16}$ & $1.1 \times 10^{-14}$ \\
\hline 20 & $1.4 \times 10^{-10}$ & $1.5 \times 10^{-04}$ & $1.1 \times 10^{-11}$ & $4.2 \times 10^{-10}$ & $2.2 \times 10^{-15}$ & $2.8 \times 10^{-13}$ \\
\hline 30 & $7.9 \times 10^{-07}$ & $8.5 \times 10^{+02}$ & $3.6 \times 10^{-09}$ & $4.3 \times 10^{-07}$ & $7.4 \times 10^{-14}$ & $1.1 \times 10^{-11}$ \\
\hline 40 & $4.7 \times 10^{-03}$ & $5.2 \times 10^{+09}$ & $2.0 \times 10^{-06}$ & $3.2 \times 10^{-04}$ & $1.4 \times 10^{-12}$ & $2.0 \times 10^{-10}$ \\
\hline 50 & $3.8 \times 10^{+01}$ & $4.3 \times 10^{+16}$ & $3.2 \times 10^{-03}$ & $7.2 \times 10^{-01}$ & $4.2 \times 10^{-11}$ & $4.6 \times 10^{-08}$ \\
\hline 60 & $5.9 \times 10^{+05}$ & $6.8 \times 10^{+23}$ & $2.7 \times 10^{+00}$ & $7.8 \times 10^{+02}$ & $1.1 \times 10^{-09}$ & $1.9 \times 10^{-07}$ \\
\hline 70 & $4.7 \times 10^{+09}$ & $5.6 \times 10^{+30}$ & $7.8 \times 10^{+02}$ & $2.1 \times 10^{+05}$ & $5.7 \times 10^{-08}$ & $7.7 \times 10^{-05}$ \\
\hline 80 & $1.4 \times 10^{+13}$ & $1.6 \times 10^{+37}$ & $1.7 \times 10^{+06}$ & $8.3 \times 10^{+09}$ & $1.0 \times 10^{-06}$ & $2.9 \times 10^{-04}$ \\
\hline 90 & $5.3 \times 10^{+16}$ & $6.5 \times 10^{+43}$ & $8.2 \times 10^{+08}$ & $8.7 \times 10^{+11}$ & $2.1 \times 10^{-05}$ & $1.5 \times 10^{-03}$ \\
\hline
\end{tabular}

$$
P_{l m}(\theta+\Delta \theta)=\sum_{k=-l}^{l} \sqrt{\frac{(l+m) !(l-k) !}{(l-m) !(l+k) !}} d_{m k}^{l}(\Delta \theta) P_{l k}(\theta) .
$$

The matrices $d^{l}$ are components of the well known Wigner $D$ matrices,

$$
D_{m k}^{l}=e^{i m \alpha} d_{m k}^{l} e^{i k \gamma}
$$

In the rotated coordinate system the translations and transformations take a more simple form and require only $O\left(p^{3}\right)$ work with regard to the length of the multipole expansions $p$.

$$
\begin{aligned}
& \omega_{l m}(\mathbf{a}+\mathbf{b})=\sum_{j=|m|}^{l} \frac{b^{l-j}}{(l-j) !} \omega_{j m}(\mathbf{a}), \\
& \mu_{l m}=\sum_{j=|m|}^{\infty} \frac{(j+l) !}{b^{j+l+1}} \omega_{j,-m}(\mathbf{a}), \quad a<b, \\
& \mu_{l m}(\mathbf{r}-\mathbf{b})=\sum_{j=l}^{\infty} \frac{b^{j-l}}{(j-l) !} \mu_{j m}(\mathbf{r}) .
\end{aligned}
$$

Vector $\mathbf{b}$ connecting the centers of the two boxes is parallel to the $Z$ axis of the rotated coordinate system,

$$
\mathbf{b}=\left(\begin{array}{c}
0 \\
0 \\
b_{z}
\end{array}\right), \quad b_{z}>0
$$

Finally, the translated multipole moments and the Taylor coefficients are rotated back using the inverse rotation matrices.
The rotation about the $Z$ axis is simply a complex multiplication. The only difficult portion is the determination of the rotation matrices $d_{m k}^{l}(\theta), 0 \leqslant \theta<2 \pi$ which corresponds to the rotation about the $Y$ axis. The nonrecursive calculation of the $d_{m k}^{l}(\theta)$ using the analytical expression requires $O\left(p^{4}\right)$ work and is numerically unstable,

$$
\begin{aligned}
d_{m k}^{l}= & \frac{1}{2^{l}} \sqrt{\frac{(l-m) !(l+m) !}{(l-k) !(l+k) !}(1+\operatorname{sgn}(k) \cos (\theta))^{|k|}} \\
& \times(\sin (\theta))^{m-|k|} \sum_{n=\max (-(m+k), 0)}^{\min (l-m, l-k)}(-1)^{l-m-n}\left(\begin{array}{c}
l-k \\
n
\end{array}\right) \\
& \times\left(\begin{array}{c}
l+k \\
l-m-n
\end{array}\right)(1+\cos (\theta))^{n}(1-\cos (\theta))^{l-m-n}, \\
& l \geqslant 0, \quad-l \leqslant k \leqslant l, \quad|k| \leqslant m \leqslant l, \\
\operatorname{sgn}(k)= & \left\{\begin{array}{c}
-1, \quad k<0 \\
0, \quad k=0 \\
1, \quad k>0,
\end{array}\right. \\
d_{m k}^{l}= & (-1)^{m+k} d_{k m}^{l}, \quad l>0, \quad-l \leqslant m<0, \\
& |m| \leqslant k \leqslant l, \quad \\
d_{m k}^{l}= & (-1)^{m+k} d_{k m}^{l}, \quad l>0, \quad 0 \leqslant m<l, \quad m<k \leqslant l,
\end{aligned}
$$

\begin{tabular}{|c|c|c|c|c|c|c|}
\hline \multirow[b]{2}{*}{$p$} & \multicolumn{2}{|c|}{ First recursion relation } & \multicolumn{2}{|c|}{ Second recursion relation } & \multicolumn{2}{|c|}{ Both recursion relations } \\
\hline & $\Delta_{d, \text { absolute }}(p)$ & $\Delta_{d, \text { relative }}(p)$ & $\Delta_{d, \text { absolute }}(p)$ & $\Delta_{d, \text { relative }}(p)$ & $\Delta_{d, \text { absolute }}(p)$ & $\Delta_{d, \text { relative }}(p)$ \\
\hline 10 & $5.7 \times 10^{-15}$ & $4.7 \times 10^{-14}$ & $4.2 \times 10^{-15}$ & $6.3 \times 10^{-14}$ & $1.0 \times 10^{-15}$ & $4.7 \times 10^{-14}$ \\
\hline 20 & $1.5 \times 10^{-13}$ & $3.7 \times 10^{-12}$ & $1.9 \times 10^{-13}$ & $8.0 \times 10^{-12}$ & $4.4 \times 10^{-15}$ & $1.0 \times 10^{-12}$ \\
\hline 30 & $3.7 \times 10^{-12}$ & $3.6 \times 10^{-10}$ & $6.5 \times 10^{-11}$ & $6.5 \times 10^{-09}$ & $4.3 \times 10^{-14}$ & $4.8 \times 10^{-11}$ \\
\hline 40 & $1.6 \times 10^{-10}$ & $9.0 \times 10^{-08}$ & $6.8 \times 10^{-09}$ & $3.4 \times 10^{-06}$ & $4.7 \times 10^{-13}$ & $1.7 \times 10^{-10}$ \\
\hline 50 & $6.7 \times 10^{-09}$ & $1.8 \times 10^{-05}$ & $7.3 \times 10^{-07}$ & $9.2 \times 10^{-03}$ & $3.3 \times 10^{-12}$ & $2.7 \times 10^{-09}$ \\
\hline 60 & $2.5 \times 10^{-07}$ & $2.9 \times 10^{-03}$ & $1.1 \times 10^{-04}$ & $9.7 \times 10^{-02}$ & $2.5 \times 10^{-11}$ & $4.2 \times 10^{-09}$ \\
\hline 70 & $8.7 \times 10^{-06}$ & $4.8 \times 10^{-01}$ & $1.9 \times 10^{-02}$ & $1.2 \times 10^{+02}$ & $5.9 \times 10^{-10}$ & $2.4 \times 10^{-08}$ \\
\hline 80 & $1.3 \times 10^{-03}$ & $4.2 \times 10^{+02}$ & $8.6 \times 10^{+00}$ & $2.5 \times 10^{+04}$ & $3.1 \times 10^{-09}$ & $2.7 \times 10^{-07}$ \\
\hline 90 & $5.0 \times 10^{-02}$ & $6.6 \times 10^{+04}$ & $1.6 \times 10^{+03}$ & $1.8 \times 10^{+06}$ & $2.7 \times 10^{-08}$ & $2.7 \times 10^{-06}$ \\
\hline
\end{tabular}

TABLE II. Maximum absolute and relative errors of the computation of the $d_{k m}^{l}(\pi / 4)$. 
TABLE III. Maximum absolute and relative errors of the computation of the $D_{k m}^{l}(\pi / 4, \pi / 4, \pi / 4)$.

\begin{tabular}{|c|c|c|c|c|c|c|}
\hline \multirow[b]{2}{*}{$p$} & \multicolumn{2}{|c|}{ First recursion relation } & \multicolumn{2}{|c|}{ Second recursion relation } & \multicolumn{2}{|c|}{ Both recursion relations } \\
\hline & $\Delta_{\mathrm{D}, \text { absolute }}(p)$ & $\Delta_{\mathrm{D}, \text { relative }}(p)$ & $\Delta_{\mathrm{D}, \text { absolute }}(p)$ & $\Delta_{\mathrm{D}, \text { relative }}(p)$ & $\Delta_{\mathrm{D}, \text { absolute }}(p)$ & $\Delta_{\mathrm{D}, \text { relative }}(p)$ \\
\hline 10 & $5.7 \times 10^{-15}$ & $4.7 \times 10^{-14}$ & $4.2 \times 10^{-15}$ & $6.3 \times 10^{-14}$ & $1.0 \times 10^{-15}$ & $4.7 \times 10^{-14}$ \\
\hline 20 & $1.5 \times 10^{-13}$ & $3.7 \times 10^{-12}$ & $1.9 \times 10^{-13}$ & $8.0 \times 10^{-12}$ & $3.6 \times 10^{-15}$ & $1.0 \times 10^{-12}$ \\
\hline 30 & $3.7 \times 10^{-12}$ & $3.6 \times 10^{-10}$ & $6.3 \times 10^{-11}$ & $6.5 \times 10^{-09}$ & $4.3 \times 10^{-14}$ & $4.8 \times 10^{-11}$ \\
\hline 40 & $1.6 \times 10^{-10}$ & $9.0 \times 10^{-08}$ & $6.8 \times 10^{-09}$ & $3.4 \times 10^{-06}$ & $4.7 \times 10^{-13}$ & $1.7 \times 10^{-10}$ \\
\hline 50 & $6.7 \times 10^{-09}$ & $1.8 \times 10^{-05}$ & $7.3 \times 10^{-07}$ & $9.2 \times 10^{-03}$ & $3.3 \times 10^{-12}$ & $2.7 \times 10^{-09}$ \\
\hline 60 & $2.5 \times 10^{-07}$ & $2.9 \times 10^{-03}$ & $1.1 \times 10^{-04}$ & $9.7 \times 10^{-02}$ & $2.5 \times 10^{-11}$ & $4.2 \times 10^{-09}$ \\
\hline 70 & $8.7 \times 10^{-06}$ & $4.8 \times 10^{-01}$ & $1.9 \times 10^{-02}$ & $1.2 \times 10^{+02}$ & $5.9 \times 10^{-10}$ & $2.4 \times 10^{-08}$ \\
\hline 80 & $1.3 \times 10^{-03}$ & $4.2 \times 10^{+02}$ & $8.4 \times 10^{+00}$ & $2.5 \times 10^{+04}$ & $3.1 \times 10^{-09}$ & $2.7 \times 10^{-07}$ \\
\hline 90 & $5.0 \times 10^{-02}$ & $6.6 \times 10^{+04}$ & $1.6 \times 10^{+03}$ & $1.8 \times 10^{+06}$ & $2.7 \times 10^{-08}$ & $2.7 \times 10^{-06}$ \\
\hline
\end{tabular}

$$
\begin{aligned}
d_{m k}^{l}= & (-1)^{m+k} d_{-m,-k}^{l}, \quad l>0, \quad-l \leqslant m<l, \\
& -l \leqslant k<-m,
\end{aligned}
$$

The essential recursion relation we will use to determine the rotation matrices is given by White and Head-Gordon ${ }^{6}$ and Edmonds. ${ }^{9}$

$$
\begin{aligned}
d_{m+1, k}^{l}= & \sqrt{\frac{l(l+1)-k(k-1)}{l(l+1)-m(m+1)}} d_{m, k-1}^{l} \\
& -\frac{(m+k)}{\sqrt{l(l+1)-m(m+1)}} \frac{\sin (\theta)}{(1+\cos (\theta))} d_{m k}^{l}, \\
& \cos (\theta) \geqslant 0, \quad l>0, \quad 0 \leqslant m<l, \quad-l<k \leqslant l,
\end{aligned}
$$

$$
\begin{gathered}
d_{m+1,-l}^{l}=\frac{(l-m)}{\sqrt{l(l+1)-m(m+1)}} \frac{\sin (\theta)}{(1+\cos (\theta))} d_{m,-l}^{l}, \\
\cos (\theta) \geqslant 0, \quad l>0, \quad 0 \leqslant m<l, \\
d_{m k}^{l}=(-1)^{m+k} d_{-m,-k}^{l}, \quad l>0, \quad-l \leqslant m<0, \\
-l \leqslant k \leqslant l .
\end{gathered}
$$

The starting point for using the recurrence is obtained by the following property of the rotation matrices:

TABLE IV. Number of floating point instructions $n_{d}(p)$ for the computation of all matrix elements $d_{k m}^{l}$ from $d_{00}^{0}$ up to $d_{p p}^{p}$.

\begin{tabular}{lc}
\hline \hline$p$ & $\begin{array}{c}\text { Number of floating } \\
\text { point instructions }\end{array}$ \\
\hline 10 & 13505 \\
20 & 96310 \\
30 & 312415 \\
40 & 725820 \\
50 & 1400525 \\
60 & 2400530 \\
70 & 3789835 \\
80 & 5632440 \\
90 & 7992345 \\
\hline \hline
\end{tabular}

$$
d_{0 k}^{l}=\left\{\begin{array}{llc}
\sqrt{\frac{(l+k) !}{(l-k) !}} P_{l,-k}, & l>0, & -l \leqslant k<0 \\
P_{l 0}, & l>0, & k=0 \\
(-1)^{k} \sqrt{\frac{(l-k) !}{(l+k) !}} P_{l k}, & l>0, & 0<k \leqslant l .
\end{array}\right.
$$

The associated Legendre polynomials $P_{l k}$ can be obtained by a stable recurrence relation,

$$
\begin{gathered}
P_{00}=1, \quad P_{l l}=(2 l-1) \sin (\theta) P_{l-1, l-1}, \quad l>0, \\
P_{l, l-1}=(2 l-1) \cos (\theta) P_{l-1, l-1}, \quad l>0, \\
P_{l k}=\frac{(2 l-1) \cos (\theta) P_{l-1, k}-(l+k-1) P_{l-2, k}}{(l-k)}, \\
\quad l>1, \quad 0 \leqslant k<l-1 .
\end{gathered}
$$

The terms $f_{l k}=\sqrt{(l-k) ! /(l+k) !}$ can be calculated numerically stable by a simple recursion,

$$
\begin{gathered}
f_{00}=1, \quad f_{l 0}=1, \quad f_{l k}=\frac{f_{l, k-1}}{\sqrt{(l-k+1)(l+k)}}, \\
l>0, \quad 0<k \leqslant l .
\end{gathered}
$$

Alternatively, the terms $f_{l k}$ can be directly included in the

TABLE V. Number of floating point instructions $n_{\mathrm{D}}(p)$ for the computation of all matrix elements $D_{k m}^{l}$ from $D_{00}^{0}$ up to $D_{p p}^{p}$.

\begin{tabular}{lc}
\hline \hline$p$ & $\begin{array}{c}\text { Number of floating } \\
\text { point instructions }\end{array}$ \\
\hline 10 & 16585 \\
20 & 113670 \\
30 & 363255 \\
40 & 837340 \\
50 & 1607925 \\
60 & 2747010 \\
70 & 4326595 \\
80 & 6418680 \\
90 & 9095265 \\
\hline \hline
\end{tabular}


TABLE VI. Maximum absolute and relative errors of the computation of the $d_{00}^{l}(\pi / 2)$

\begin{tabular}{rll}
\hline \hline & \multicolumn{2}{c}{ Both recursion relations } \\
\cline { 2 - 3 }$p$ & $\Delta_{d, \text { absolute }}(p)$ & $\Delta_{d, \text { relative }}(p)$ \\
\hline 10 & $0.0 \times 10^{+00}$ & $0.0 \times 10^{+00}$ \\
20 & $0.0 \times 10^{+00}$ & $0.0 \times 10^{+00}$ \\
30 & $0.0 \times 10^{+00}$ & $0.0 \times 10^{+00}$ \\
40 & $0.0 \times 10^{+00}$ & $0.0 \times 10^{+00}$ \\
50 & $0.0 \times 10^{+00}$ & $0.0 \times 10^{+00}$ \\
60 & $0.0 \times 10^{+00}$ & $0.0 \times 10^{+00}$ \\
70 & $8.7 \times 10^{-18}$ & $9.1 \times 10^{-17}$ \\
80 & $2.1 \times 10^{-17}$ & $2.3 \times 10^{-16}$ \\
90 & $2.9 \times 10^{-17}$ & $3.4 \times 10^{-16}$ \\
100 & $3.8 \times 10^{-17}$ & $4.7 \times 10^{-16}$ \\
200 & $3.9 \times 10^{-17}$ & $5.3 \times 10^{-16}$ \\
300 & $3.9 \times 10^{-17}$ & $5.3 \times 10^{-16}$ \\
400 & $3.9 \times 10^{-17}$ & $7.0 \times 10^{-16}$ \\
500 & $3.9 \times 10^{-17}$ & $7.0 \times 10^{-16}$ \\
600 & $3.9 \times 10^{-17}$ & $7.0 \times 10^{-16}$ \\
700 & $3.9 \times 10^{-17}$ & $9.4 \times 10^{-16}$ \\
800 & $3.9 \times 10^{-17}$ & $9.4 \times 10^{-16}$ \\
900 & $3.9 \times 10^{-17}$ & $9.4 \times 10^{-16}$ \\
1000 & $3.9 \times 10^{-17}$ & $9.4 \times 10^{-16}$ \\
\hline \hline
\end{tabular}

recursion of the associated Legendre polynomials. The scaled associated Legendre polynomials $\widetilde{P}_{l k}=f_{l k} P_{l k}, l \geqslant 0, m$ $\leqslant 0 \leqslant l$ can also be calculated numerically stable,

$\tilde{P}_{00}=1, \quad \widetilde{P}_{l l}=\sqrt{\frac{2 l-1}{2 l}} \sin (\theta) \tilde{P}_{l-1, l-1}, \quad l>0$,

$\widetilde{P}_{l, l-1}=\sqrt{2 l-1} \cos (\theta) \tilde{P}_{l-1, l-1}, \quad l>0$,

$\tilde{P}_{l k}=\frac{(2 l-1) \cos (\theta) \tilde{P}_{l-1, k}-\sqrt{(l-k-1)(l+k-1)} \tilde{P}_{l-2, k}}{\sqrt{(l-k)(l+k)}}$,

$l>1, \quad 0 \leqslant k<l-1$.

Unfortunately, Eqs. (18) and (19) become unstable in case of higher moments. We have combined Eqs. (18) and (19) with a second recurrence to overcome the numerical instabilities,

$$
\begin{aligned}
d_{m, k-1}^{l}= & \sqrt{\frac{l(l+1)-m(m+1)}{l(l+1)-k(k-1)}} d_{m+1, k}^{l} \\
& +\frac{(m+k)}{\sqrt{l(l+1)-k(k-1)}} \frac{\sin (\theta)}{(1+\cos (\theta))} d_{m k}^{l}, \\
& \cos (\theta) \geqslant 0, \quad l>0, \quad 0 \leqslant m<l, \quad-l<k \leqslant l,
\end{aligned}
$$

$$
\begin{array}{r}
d_{l, k-1}^{l}=\frac{(l+k)}{\sqrt{l(l+1)-k(k-1)}} \frac{\sin (\theta)}{(1+\cos (\theta))} d_{l k}^{l}, \\
\cos (\theta) \geqslant 0, \quad l>0, \quad-l<k \leqslant l,
\end{array}
$$

TABLE VII. Maximum absolute and relative errors of the computation of the $d_{00}^{l}(\pi / 4)$.

\begin{tabular}{rrr}
\hline \hline & \multicolumn{2}{c}{ Both recursion relations } \\
\cline { 2 - 3 }$p$ & $\Delta_{d, \text { absolute }}(p)$ & $\Delta_{d, \text { relative }}(p)$ \\
\hline 10 & $2.8 \times 10^{-16}$ & $2.2 \times 10^{-15}$ \\
20 & $2.8 \times 10^{-16}$ & $3.1 \times 10^{-15}$ \\
30 & $3.5 \times 10^{-16}$ & $5.2 \times 10^{-15}$ \\
40 & $3.9 \times 10^{-16}$ & $6.4 \times 10^{-15}$ \\
50 & $4.2 \times 10^{-16}$ & $8.1 \times 10^{-15}$ \\
60 & $4.7 \times 10^{-16}$ & $9.7 \times 10^{-15}$ \\
70 & $5.2 \times 10^{-16}$ & $1.2 \times 10^{-14}$ \\
80 & $5.5 \times 10^{-16}$ & $1.4 \times 10^{-14}$ \\
90 & $5.8 \times 10^{-16}$ & $1.5 \times 10^{-14}$ \\
100 & $6.9 \times 10^{-16}$ & $1.9 \times 10^{-14}$ \\
200 & $8.5 \times 10^{-16}$ & $3.3 \times 10^{-14}$ \\
300 & $1.0 \times 10^{-15}$ & $4.9 \times 10^{-14}$ \\
400 & $1.2 \times 10^{-15}$ & $6.4 \times 10^{-14}$ \\
500 & $1.3 \times 10^{-15}$ & $8.1 \times 10^{-14}$ \\
600 & $1.4 \times 10^{-15}$ & $9.5 \times 10^{-14}$ \\
700 & $1.5 \times 10^{-15}$ & $1.1 \times 10^{-13}$ \\
800 & $1.7 \times 10^{-15}$ & $1.3 \times 10^{-13}$ \\
900 & $1.8 \times 10^{-15}$ & $1.5 \times 10^{-13}$ \\
1000 & $1.9 \times 10^{-15}$ & $1.6 \times 10^{-13}$ \\
\hline \hline & & \\
$d_{m k}^{l}(-1)^{m+k} d_{-m,-k}^{l}, \quad l>0$, & \\
& & \\
\hline
\end{tabular}

The starting point for using this recurrence is obtained by Eq. (13) for $k=l$,

$$
\begin{aligned}
& d_{m l}^{l}=(-1)^{l+m} g_{l m}(1+\cos (\theta))^{m}(\sin (\theta))^{l-m}, \\
& g_{l m}=\frac{1}{2^{l}} \sqrt{\frac{(2 l) !}{(l-m) !(l+m) !}}, \quad l>0, \quad 0 \leqslant m \leqslant l .
\end{aligned}
$$

The terms $g_{l m}$ can be computed by the following numerically stable recursion:

$$
\begin{aligned}
& g_{00}=1, \quad g_{l 0}=\sqrt{\frac{2 l-1}{2 l}} g_{l-1,0}, \quad l>0, \\
& g_{l m}=\sqrt{\frac{l-m+1}{l+m}} g_{l, m-1}, \quad l>0, \quad 0<m \leqslant l .
\end{aligned}
$$

In addition to the two recurrences the error accumulations are evaluated for both of the recurrences by summations of the absolute values of all terms in the recursion relations to decide which recurrence is more accurate for a given element of the rotation matrix. Both of the recursion relations should be used only for $\cos (\theta) \geqslant 0$. In case of $\cos (\theta)<0$ addition theorems can be used given by Edmonds. ${ }^{9}$ The recursion relations are needed only for $0<\theta \leqslant \pi / 2$, 


$$
d_{m k}^{l}(\theta)=\left\{\begin{array}{l}
\delta_{m k}, \quad \theta=0 \\
(-1)^{l+k} d_{-m, k}^{l}(\pi-\theta)=(-1)^{l+m} d_{m,-k}^{l}(\pi-\theta), \quad \frac{\pi}{2}<\theta<\pi \\
(-1)^{l+k} \delta_{-m, k}=(-1)^{l+k} \delta_{m,-k}, \quad \theta=\pi \\
(-1)^{l+k} d_{m,-k}^{l}(\theta-\pi)=(-1)^{l+m} d_{-m, k}^{l}(\theta-\pi), \quad \pi<\theta<\frac{3 \pi}{2} \\
(-1)^{m+k} d_{m k}^{l}(2 \pi-\theta), \quad \frac{3 \pi}{2} \leqslant \theta<2 \pi .
\end{array}\right.
$$

\section{RESULTS}

All calculations were performed in 64 bits accuracy using the ANSI/IEEE standard for the floating point representation. The combination of the two recurrences shows a significant improvement of accuracy. Table I shows the maximum absolute and relative errors for $\theta=\pi / 2$, Table II for $\theta=\pi / 4$. The computations of all elements of the rotation matrices in sufficient accuracy are done with MAPLE. ${ }^{10,11}$

$$
\Delta_{d, \text { absolute }}(p)=\max _{0 \leqslant l \leqslant p,-l \leqslant m \leqslant l,-l \leqslant k \leqslant l}\left|d_{m k, \text { computed }}^{l}-d_{m k, \text { exact }}^{l}\right|,
$$

$$
\Delta_{d, \text { relative }}(p)=\max _{0 \leqslant l \leqslant p,-l \leqslant m \leqslant l,-l \leqslant k \leqslant l}\left|\frac{d_{m k, \text { computed }}^{l}-d_{m k, \text { exact }}^{l}}{d_{m k, \text { exact }}^{l}}\right| \text {. }
$$

Table III shows the maximum absolute and relative errors for the complete Wigner $D$ matrix for all three Euler angles equal to $\pi / 4$.

$$
D_{m k}^{l}\left(\frac{\pi}{4}, \frac{\pi}{4}, \frac{\pi}{4},\right)=e^{i m(\pi / 4)} d_{m k}^{l}\left(\frac{\pi}{4}\right) e^{i k(\pi / 4)} .
$$

With respect to the absolute and relative errors the maximum of the real and imaginary parts of $D_{m k}^{l}$ are taken, ${ }^{12}$

$$
\begin{aligned}
\Delta_{D, \text { absolute }}(p)= & \max \left(\Delta_{R, \text { absolute }}(p), \Delta_{I, \text { absolute }}(p)\right), \\
\Delta_{R, \text { absolute }}(p)= & \max _{0 \leqslant l \leqslant p,-l \leqslant m \leqslant l,-l \leqslant k \leqslant l} \mid \operatorname{Re}\left(d_{m k, \text { computed }}^{l}\right) \\
& -\operatorname{Re}\left(d_{m k, \text { exact }}^{l}\right) \mid,
\end{aligned}
$$

$$
\begin{aligned}
\Delta_{I, \text { absolute }}(p)= & \max _{0 \leqslant l \leqslant p,-l \leqslant m \leqslant l,-l \leqslant k \leqslant l} \mid \operatorname{Im}\left(d_{m k, \text { computed }}^{l}\right) \\
& -\operatorname{Im}\left(d_{m k, \text { exact }}^{l}\right) \mid,
\end{aligned}
$$

$$
\begin{aligned}
& \Delta_{D, \text { relative }}(p)=\max \left(\Delta_{R, \text { relative }}(p), \Delta_{I, \text { relative }}(p)\right), \\
& \Delta_{R, \text { relative }}(p)=\max _{0 \leqslant l \leqslant p,-l \leqslant m \leqslant l,-l \leqslant k \leqslant l}\left|\frac{\operatorname{Re}\left(D_{m k, \text { computed }}^{l}\right)-\operatorname{Re}\left(D_{m k, \text { exact }}^{l}\right)}{\operatorname{Re}\left(D_{m k \text {,exact }}^{l}\right)}\right|, \\
& \Delta_{I, \text { relative }}(p)=\max _{0 \leqslant l \leqslant p,-l \leqslant m \leqslant l,-l \leqslant k \leqslant l}\left|\frac{\operatorname{Im}\left(D_{m k, \text { computed }}^{l}\right)-\operatorname{Im}\left(D_{m k, \text { exact }}^{l}\right)}{\operatorname{Im}\left(D_{m k, \text { exact }}^{l}\right)}\right| .
\end{aligned}
$$

The absolute errors are significant less compared to the work of Choi et al. ${ }^{13}$ Unfortunately, the authors have reported only absolute errors up to the the matrix element $D_{40,40}^{40}$. Already for $p=20$ our results are about three magnitudes more accurate. For $p=30$ and $p=40$ we have obtained five and six, respectively, significant digits more. For $p=90$ we have still six correct digits in the mantissa.

The Tables IV and V show the numbers of floating point instructions for the computation of both the martices $D$ and $d$. The floating point instructions are counted for the compu- tation of all matrix elements, from the elements $d_{00}^{0}$ and $D_{00}^{0}$ up to the elements $d_{p p}^{p}$ and $D_{p p}^{p}$. The numbers of floating point instructions depend cubically on $p$. The values in the Tables IV and V can be computed by polynomials of third degree in $p$. These polynomials are the results of symbolic summation, ${ }^{10,11}$

$$
n_{d}(p)=\frac{64 p^{3}+159 p^{2}+113 p}{6}
$$




$$
n_{D}(p)=\frac{24 p^{3}+85 p^{2}+67 p}{2} .
$$

All calculations run on the IBM p690 cluster at the Research Centre Jülich. On an IBM Power4+processor $(1.7 \mathrm{GHz})$ code of this type performs at roughly 1.7 Gflops. For example, the calculation of the first matrix elements $d_{m k}^{l}\left(d_{00}^{0}\right.$ up to $\left.d_{10,10}^{10}\right)$ requires approximately $8 \mathrm{~ms}$. The computation time for the evaluation of the Wigner rotation matrices within the FMM is negligible.

An important criterion for the numerical stability is the accuracy of the calculation of the matrix elements $d_{00}^{l}$ for the angles $\pi / 2$ and $\pi / 4$. We define again maximum absolute and relative errors,

$$
\begin{aligned}
& \Delta_{d, \text { absolute }}(p)=\max _{0 \leqslant l \leqslant p}\left|d_{00, \text { computed }}^{l}-d_{00, \text { exact }}^{l}\right|, \\
& \Delta_{d, \text { relative }}(p)=\max _{0 \leqslant l \leqslant p}\left|\frac{d_{00, \text { computed }}^{l}-d_{00, \text { exact }}^{l}}{d_{00, \text { exact }}^{l}}\right| .
\end{aligned}
$$

The relative errors of our approach are in the range of the machine error of the ANSI/IEEE floating point representa- tion of $2^{-52} \approx 2.2 \times 10^{-16}$ for the angle $\pi / 2$. The relative errors for the angle $\pi / 4$ are greater compared to the angle $\pi / 2$ because the recursions are less accurate (Tables VI and VII).

${ }^{1}$ M. P. Allen and D. J. Tildesley, Computer Simulation of Liquids (Oxford University, Oxford, 1990).

${ }^{2}$ J. M. Dawson, Rev. Mod. Phys. 55, 403 (1983).

${ }^{3}$ L. F. Greengard, The Rapid Evaluation of Potential Fields in Particle Systems (MIT, Cambridge, 1988).

${ }^{4}$ L. F. Greengard and V. Rokhlin, J. Comput. Phys. 60, 187 (1985).

${ }^{5}$ J. M. Ugalde and C. Sarasola, Int. J. Quantum Chem. 62, 273 (1997).

${ }^{6}$ C. A. White and M. Head-Gordon, J. Chem. Phys. 105, 5061 (1996).

${ }^{7}$ W. D. Elliott and J. A. Board, Jr., SIAM J. Sci. Comput. (USA) 17, 398 (1996)

${ }^{8}$ C. A. White and M. Head-Gordon, J. Chem. Phys. 101, 6593 (1994).

${ }^{9}$ A. R. Edmonds, Angular Momentum in Quantum Mechanics (Princeton University Press, Princeton, 1957).

${ }^{10}$ Maple 9 Learning Guide (Maplesoft, Toronto, 2003).

${ }^{11}$ M. B. Monagan, K. O. Geddes, K. M. Heal, G. Labahn, S. M. Vorkoetter, J. McCarron, and P. DeMarco, Maple 9 Introductory Programming Guide (Maplesoft, Toronto, 2003).

${ }^{12}$ I. J. Davis, Comput. J. 35, 636 (1992).

${ }^{13}$ C. H. Choi, J. Ivanic, M. S. Gordon, and K. Ruedenberg, J. Chem. Phys. 111, 8825 (1999). 